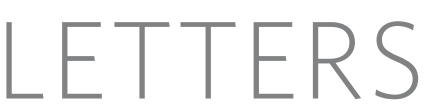

\title{
The pectoral fin of Panderichthys and the origin of digits
}

\author{
Catherine A. Boisvert ${ }^{1}$, Elga Mark-Kurik ${ }^{2}$ \& Per E. Ahlberg ${ }^{1}$
}

One of the identifying characteristics of tetrapods (limbed vertebrates) is the presence of fingers and toes. Whereas the proximal part of the tetrapod limb skeleton can easily be homologized with the paired fin skeletons of sarcopterygian (lobe-finned) fish, there has been much debate about the origin of digits. Early hypotheses ${ }^{1}$ interpreted digits as derivatives of fin radials, but during the 1990s the idea gained acceptance that digits are evolutionary novelties without direct equivalents in fish fin skeletons. This was partly based on developmental genetic data ${ }^{2}$, but also substantially on the pectoral fin skeleton of the elpistostegid (transitional fish/ tetrapod) Panderichthys, which appeared to lack distal digit-like radials $s^{3}$. Here we present a CT scan study of an undisturbed pectoral fin of Panderichthys demonstrating that the plate-like 'ulnare' of previous reconstructions is an artefact and that distal radials are in fact present. This distal portion is more tetrapod-like than that found in Tiktaalik ${ }^{4}$ and, in combination with new data about fin development in basal actinopterygians ${ }^{5}$, sharks $^{6}$ and lungfish ${ }^{7}$, makes a strong case for fingers not being a novelty of tetrapods but derived from pre-existing distal radials present in all sarcopterygian fish.

A near-complete specimen of Panderichthys from the late Middle Devonian period (385 million years ago; see Supplementary Information) of Lode, Latvia (Institute of Geology at Tallinn University of Technology specimen number GIT434-1) forms the basis of this study. Although the dorsal part of the skull and left side of the body have suffered substantial damage from a mechanical excavator, the specimen was originally well preserved. Notably, its body axis is straight (determined from the alignment of dorsal midline scales and the symmetry plane of the skull) and it appears less dorsoventrally compressed than others from the same locality. This is shown, for example, by the narrow skull outline and near-vertical cheek-plate fragments, distinctly different from the flattened and splayed skulls that have been published ${ }^{8,9}$. The specimen also contains the only known pelvis and pelvic fin skeleton of Panderichthys ${ }^{10}$.

The right pectoral fin and most of the shoulder girdle are preserved in articulation, with the fin concealed under the body. This region of the specimen was CT scanned at the East-Tallinn Central Hospital (see Methods Summary and Supplementary Methods). The scanned region comprises the entire fin endoskeleton and an estimated $40 \%$ of the lepidotrichial fin web, as well as most of the shoulder girdle. The presence of X-ray-reflective crystal growths in the shoulder region unfortunately prevented complete modelling of the scapulocoracoid. The entire fin is covered by scales and lepidotrichia, which cannot be modelled individually but are easily separated from the darker endoskeleton. The leading or preaxial margin of the fin is dipping ventrolaterally into the substrate (Fig. 1a-c). In contrast to early tetrapods, in which the limb projects at an angle from the body wall $^{11}$, the fin of Panderichthys is oriented anteroposteriorly in line with the body axis. In distal cross section (Fig. 1c), the fin is slightly cambered, convex dorsally and concave ventrally. The dorsal part of the cleithrum is missing but the notably long anocleithrum is complete (Fig. 1b, d). The proximal quarter of the anocleithrum is overlapped by the supracleithrum.

The fin endoskeleton is complete and is composed of the humerus, radius, ulna, ulnare, bipartite intermedium and four small distal radials arranged in a transverse terminal array (Fig. 2). The humerus closely resembles the most recent published description ${ }^{12}$ as far as the morphological details are concerned, but it is much less flattened, displaying a pear-shaped proximal cross section rather than the

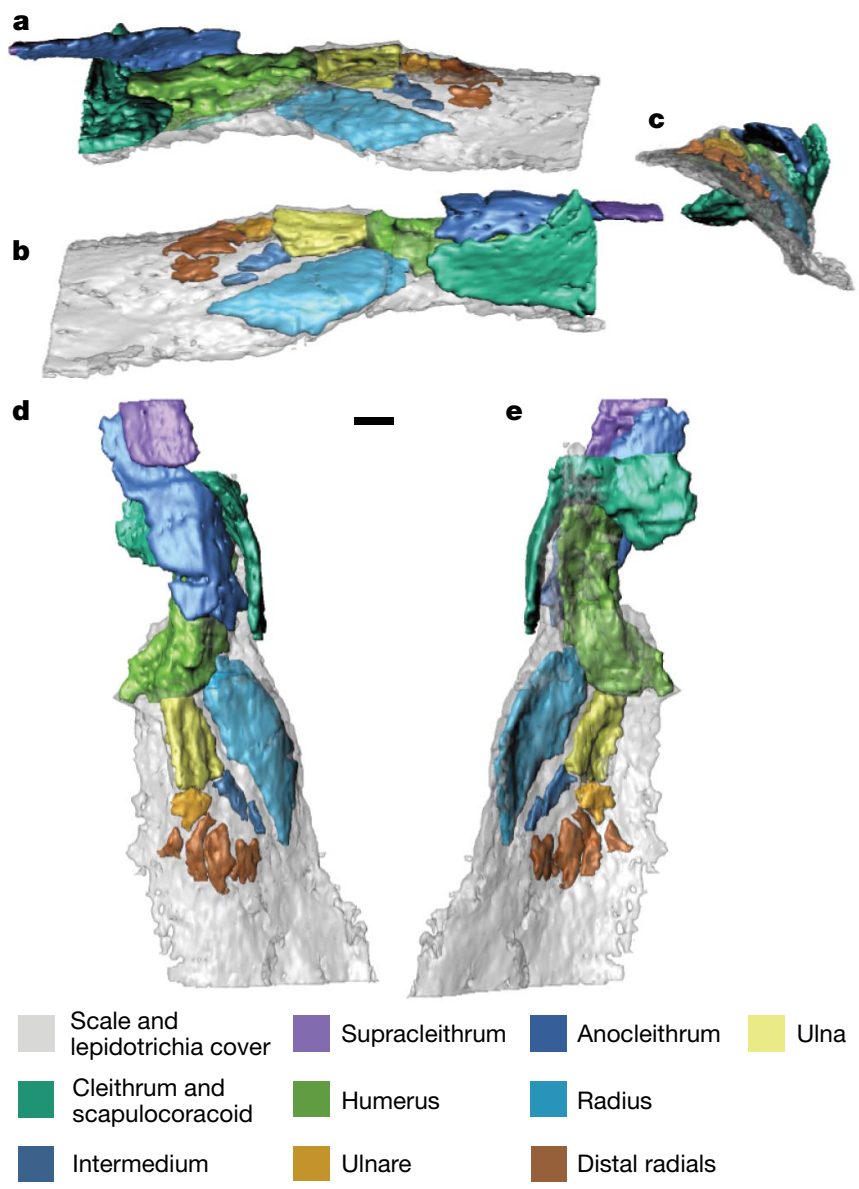

Figure 1 | Pectoral fin, girdle and scale plus lepidotrichia cover of Panderichthys. a, Postaxial view; b, preaxial view; c, distal cross section; d, dorsal view; and e, ventral view. Scale bar, $1 \mathrm{~cm}$. 
reported very dorsoventrally flattened shape ${ }^{13}$. We attribute this difference to the unusually three-dimensional preservation of GIT434-1. In most respects, the humerus agrees well with that of Tiktaalik ${ }^{4}$. However, the orientation of the scapulohumeral joint is
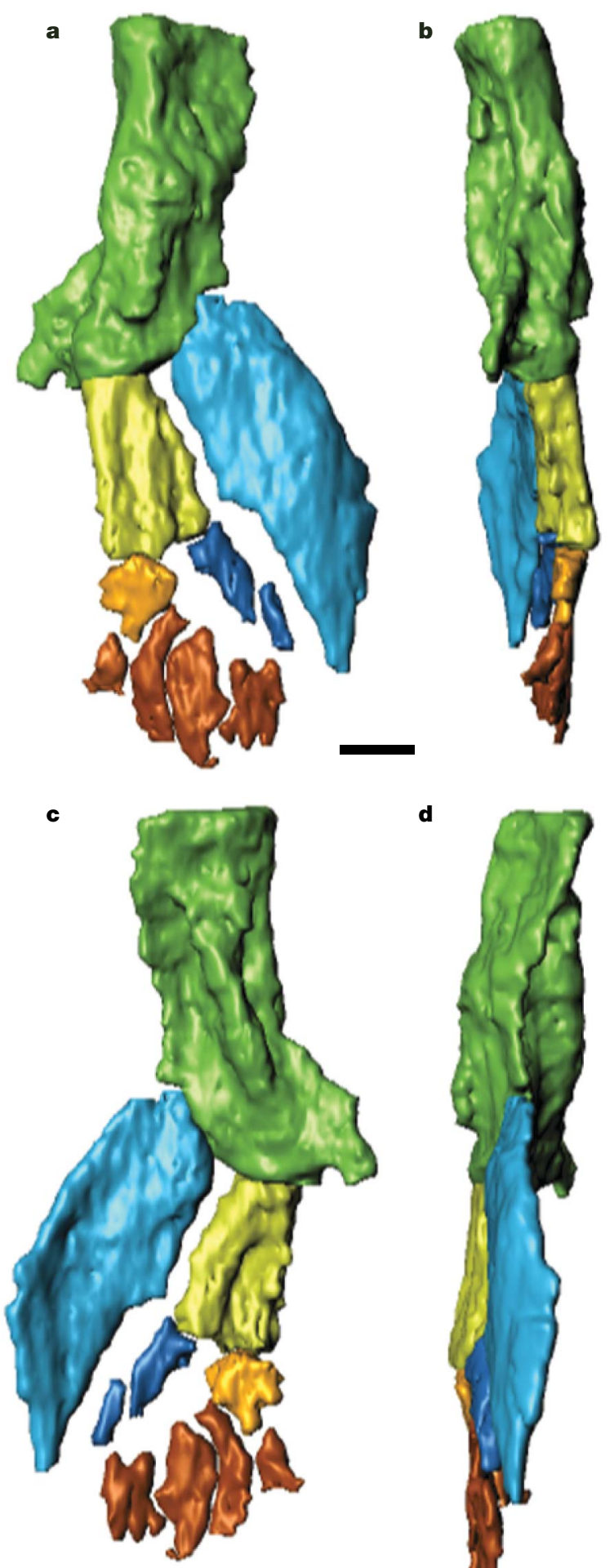

d
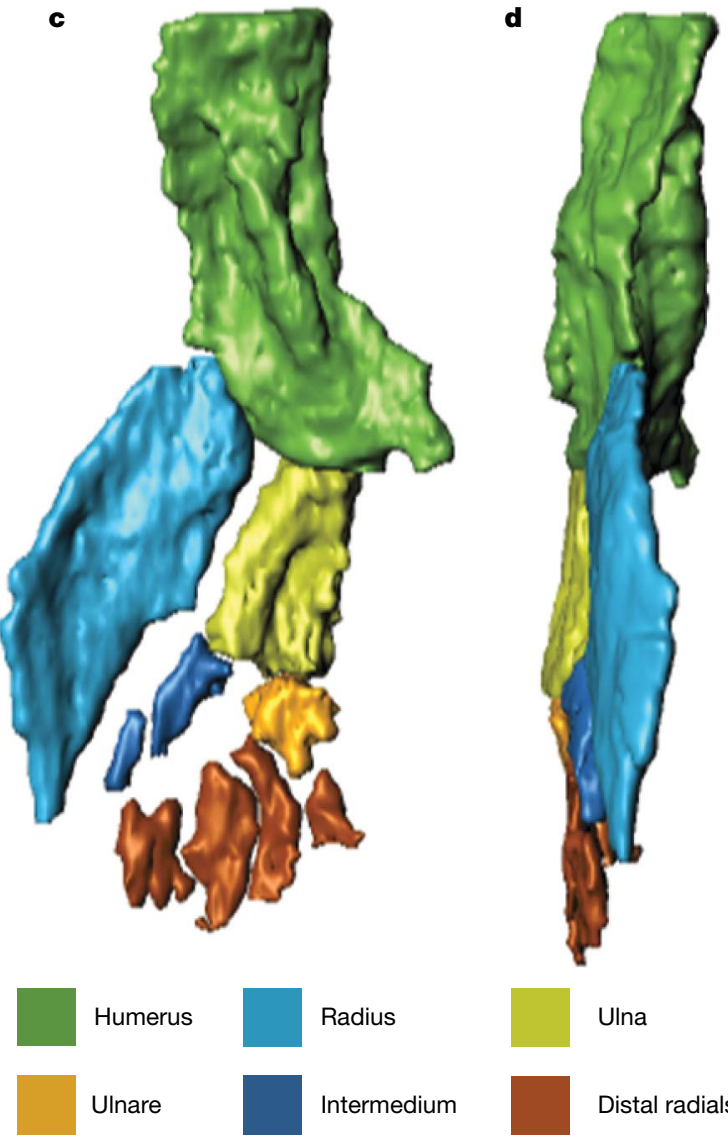

Ulna

Distal radials

Figure 2 | Endoskeleton of the pectoral fin of Panderichthys rhombolepis. a, Dorsal; b, postaxial; c, ventral; and d, preaxial views. Scale bar, $1 \mathrm{~cm}$. parallel to the scan plane, and the thickness of scan slices $(0.6 \mathrm{~mm})$ prevents us from reconstructing the joint surfaces in detail.

The ectepicondyle is linear, low and parallel to the post-axial edge of the humerus (Fig. 2a). It is shorter than in Tiktaalik ${ }^{4}$, ending some distance from the ulnar articulation. On the ventral surface, the humeral ridge is slightly oblique (Fig. 2b) and much higher than previously described ${ }^{12}$, terminating at the base of the entepicondyle rather than extending onto its ventral face as in Tiktaalik $k^{4,13}$. The radius is a sickle-shaped blade, very slender in axial aspect and convex ventrally. It is smooth on both the dorsal and the ventral surfaces and has a thickened lateral edge. Like in Tiktaalik ${ }^{4}$, the ulna has a complex shape with several longitudinal grooves and ridges.

The most distal portion of the fin endoskeleton had been described as being composed of only two elements-a large plate-like ulnare and a narrow intermedium in close contact ${ }^{3}$ — but examination by CAB of the published material (Palaeontological Institute of the Russian Academy of Sciences, Moscow specimen numbers PIN 3547/26 and PIN 3547/18) showed that the region is covered by a thin sediment film bearing impressions of the inner faces of scales, which conceals any endoskeletal elements underneath. These scale impressions can in fact be discerned in the published reconstruction drawings as a regular pattern on the 'ulnare's. Our CT scan thus reveals the distal fin endoskeleton of Panderichthys for the first time. The ulnare is small and flattened, articulating proximally with the ulna and distally with two terminal radials. The intermedium is a flattened rod, narrower than previously described, articulating directly in line to the lateral ridge of the ulna. It appears to consist of separate proximal and distal components as in Tiktaalik ${ }^{4}$, but the resolution of the 'joint' is poor and it is possible that this divide is an artefact.

The distal-most part of the fin is composed of four distal radials, arranged in a slight arc distal to the ulnare and the intermedium. The separation of radials from each other and from the ulnare can be seen by density differences in the scan (see Supplementary Information) but also through slight differences in the orientation of the elements. All radials have flattened cross-sections of the same thickness but they vary in width. If the axis of the fin is interpreted to extend through the middle of the ulnare, one of the distal radials is postaxial, one is in line with the ulnare and two are preaxial. The two most preaxial distal radials do not articulate directly with an axial element (Fig. 2).

Our reinterpretation of the distal fin endoskeleton of Panderichthys removes the final piece of evidence supporting the formerly popular hypothesis that tetrapod digits are wholly new structures without homologues in sarcopterygian fish fins ${ }^{2,14-16}$. This hypothesis, which was based partly on the complete absence of plausible digit homologues in Panderichthys (then the closest known relative of tetrapods $)^{3}$, has already been called into question by the discovery of digit-like radials in Tiktaalik ${ }^{4}$ and the fact that Hox gene expression patterns closely resembling those associated with digit formation in tetrapods occur in the distal fin skeletons of paddlefish $^{5}$ and Australian lungfish ${ }^{7}$. Our new data show that Panderichthys is not an anomaly: like Tiktaalik and other fish members of the Tetrapodomorpha ${ }^{17,18}$ it has distal radials that can be interpreted as digit homologues.

The pectoral fin skeletons of Panderichthys and Tiktaalik share certain unusual features such as a blade-like radius and a longitudinal ridge-and-groove on the flexor surface of the ulna. These can tentatively be interpreted as attributes of the 'elpistostegid' segment of the tetrapod stem lineage and thus ancestral for the tetrapod forelimb. Given that recent phylogenies consistently place Panderichthys below Tiktaalik in the tetrapod stem group ${ }^{19,20}$, it is surprising to discover that its pectoral fin skeleton is more limb-like than that of its supposedly more derived relative. In Tiktaalik', like in 'osteolepiforms'17 and rhizodonts ${ }^{18}$ (more primitive fish members of the stem group), the ulna and ulnare are of similar size. The axis of the fin comprises two more elements distal to the ulnare, and the distal radials are arranged pinnately around this axis. In contrast, in Panderichthys and tetrapods, the ulna is much longer than the ulnare, the ulnare 

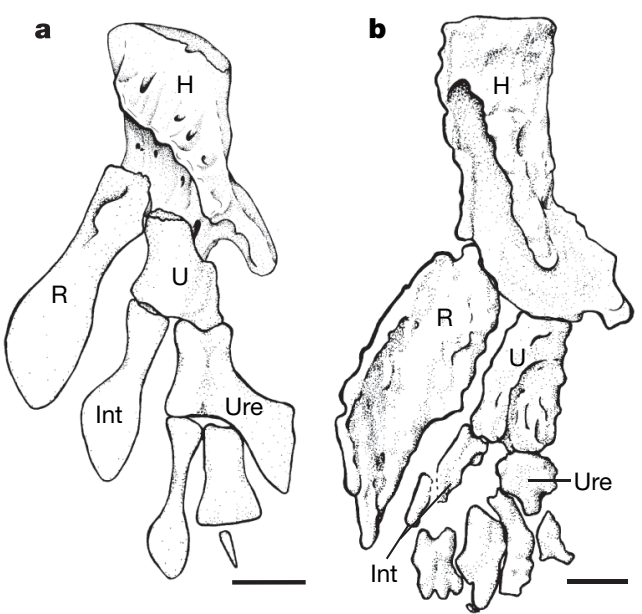

Figure 3 | Pectoral endoskeleton of tetrapodomorph fish and a tetrapod in ventral view. Pectoral fins of: a, Eusthenopteron; b, Panderichthys; and c, Tiktaalik. d, Limb of Acanthostega. H, humerus; Int, intermedium;

is the last axial element, and the distal radials/digits are arranged in a transverse fan shape $e^{11,21}$ (Fig. 3). It is difficult to say whether this character distribution implies that Tiktaalik is autapomorphic, that Panderichthys and tetrapods are convergent, or that Panderichthys is closer to tetrapods than Tiktaalik. At any rate, it demonstrates that the fish-tetrapod transition was accompanied by significant character incongruence in functionally important structures.

\section{METHODS SUMMARY}

The specimen was CT scanned on a medical scanner at East-Tallinn Central Hospital, Estonia, and modelled independently using Mimics v.9.11 (Materialise NV, http://www.materialise.com/mimics) and rendered using Rhinoceros (version 3.0 SR4) with the plug-in Bongo (version 1.0).

Received 12 June; accepted 14 August 2008. Published online 21 September 2008.

1. Gegenbaur, C. Grundriss der vergleichenden anatomie (Wilhelm Engelmann, 1874).

2. Sordino, P., van der Hoeven, F. \& Duboule, D. Hox gene expression in teleost fins and the origin of vertebrate digits. Nature 375, 678-681 (1995).

3. Vorobyeva, E. I. The role of development and function in formation of tetrapodlike pectoral fins. Zh. Obshch. Biol. 53, 149-158 (1992).

4. Shubin, N. H., Daeschler, E. B. \& Jenkins, F. A. Jr. The pectoral fin of Tiktaalik roseae and the origin of the tetrapod limb. Nature 440, 764-771 (2006).

5. Davis, M. C., Dahn, R. D. \& Shubin, N. H. An autopodial-like pattern of Hox expression in the fins of a basal actinopterygian fish. Nature 447, 473-476 (2007).

6. Dahn, R. D. et al. Sonic hedgehog function in chondrichthyan fins and the evolution of appendage patterning. Nature 445, 311-314 (2007)

7. Johanson, Z. et al. Fish fingers: digit homologues in sarcopterygians fish fins. J. Exp. Zool. (Mol. Dev. Evol.) 308B, 757-768 (2007).

8. Vorobyeva, E. I. Observations on two rhipidistian fishes from the Upper Devonian of Lode, Latvia. Zool. J. Linn. Soc. 70, 191-201 (1980).

9. Vorobyeva, E. I. \& Schultze, H.-P. in Origins of the Higher Groups of Tetrapods: Controversy and Consensus (eds Schultze, H.-P. \& Trueb L.) 68-109 (Cornell Univ. Press, 1991)

10. Boisvert, C. A. The pelvic fin and girdle of Panderichthys and the origin of tetrapod locomotion. Nature 438, 1145-1147 (2005).

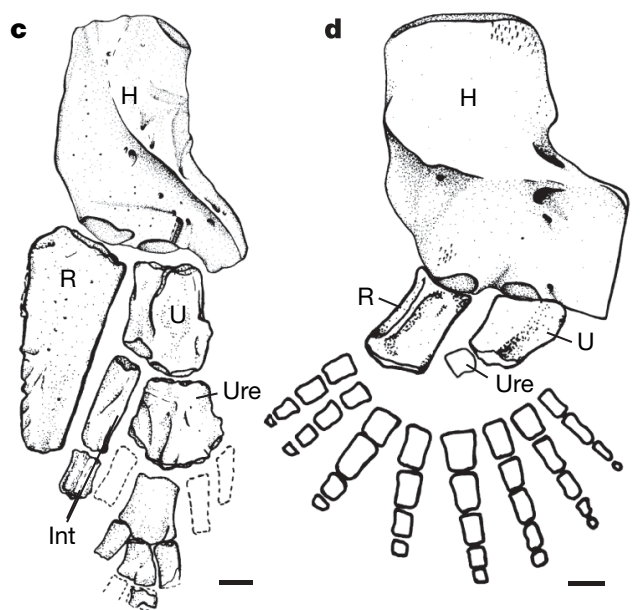

$R$, radius; $U$, ulna; Ure, ulnare. Scale bar, $1 \mathrm{~cm}$. a is redrawn from ref. 17, c from ref. 4 and $\mathrm{d}$ from ref. 11 .

11. Coates, M. I. The Devonian tetrapod Acanthostega gunnari Jarvik: postcranial anatomy, basal interrelationships and patterns of skeletal evolution. Trans. R. Soc. Edinb. Earth Sci. 87, 363-421 (1996).

12. Vorobyeva, E. I. Morphology of the humerus in the rhipidistian Crossopterygii and the origin of tetrapods. Paleontol. J. 34, 632-641 (2000).

13. Boisvert, C. A. in Forty Years of Early Vertebrates: Papers from the 11th International Symposium on Early and Lower Vertebrates (eds Ahlberg, P. E., Blom H. \& Boisvert, C. A.) (Vol. Acta Zool. special volume, Blackwell, in the press).

14. Shubin, N. H., Tabin, C. \& Carroll, S. Fossils, genes and the evolution of animal limbs. Nature 388, 639-648 (1997).

15. Gregory, W. K. \& Raven, H. C. Studies on the origin and early evolution of paired fins and limbs. Ann. NY Acad. Sci. 42, 273-360 (1941)

16. Westoll, T. S. The origin of the primitive tetrapod limb. Proc. R. Soc. Lond. B 131, 373-393 (1943).

17. Andrews, M. S. \& Westoll, S. T. The postcranial skeleton of Eusthenopteron foordi Whiteaves. Trans. R. Soc. Edinb. 68, 207-328 (1970).

18. Davis, M. C., Shubin, N. H. \& Daeschler, E. B. A new specimen of Sauripterus taylori (Sarcopterygii, Osteichthyes) from the Famennian Catskill Formation of North America. J. Vert. Paleontol. 24, 26-40 (2004).

19. Daeschler, E. B., Shubin, N. H. \& Jenkins, F. A. Jr. A Devonian tetrapod-like fish and the evolution of the tetrapod body plan. Nature 440, 757-763 (2006).

20. Long, J. A. et al. An exceptional Devonian fish from Australia sheds light on tetrapod origins. Nature 444, 199-202 (2006).

21. Jarvik, E. The Devonian tetrapod Ichthyostega. Fossils and Strata 40, 1-213 (1996).

Supplementary Information is linked to the online version of the paper at www.nature.com/nature.

Acknowledgements We thank P. Ross and the team at East-Tallinn Central Hospital, Estonia for the CT-scanning, E. Vorobyeva for permission to examine the Panderichthys material in her care, N. Shubin for access to Tiktaalik, and M. I. Coates for valuable discussions. C.A.B. and P.E.A. are supported by Vetenskapsrådet.

Author Contributions E.M.-K wrote one of the Supplementary Information files and arranged the logistics for the CT scan, P.E.A. and E.M.-K. scanned the specimen, and C.A.B and P.E.A. produced the models and descriptions.

Author Information Reprints and permissions information is available at www.nature.com/reprints. Correspondence and requests for materials should be addressed to C.A.B. (catherine.boisvert@ebc.uu.se). 